\title{
Síndrome del desfiladero cervicotorácico unilateral en un caso con costillas cervicales bilaterales, ¿siempre produce compresión?
}

\author{
Unilateral thoracic outlet syndrome in a case with bilateral cervical ribs, does it always \\ produce compression?
}

Juan M. Alemán-Iñiguez ${ }^{*}$ y Hernán Hermida-Córdova²

'Departamento de Neurocirugía, Universidad San Francisco de Quito, Quito; ²Departamento de Cirugía de Tórax, Universidad de Cuenca, Cuenca. Ecuador

\begin{abstract}
Resumen
Antecedentes: La costilla cervical puede ser un raro hallazgo en sujetos asintomáticos. Cuando produce compresión, afecta variablemente a las estructuras vasculonerviosas del plexo braquial. El cuadro clínico es progresivo. Los estudios de imagen indican el sitio de conflicto osteoneurovascular, y los electrofisiológicos, el grado de afección neural. Caso clínico: Presentamos un paciente con historia de 8 meses de paresia y disestesias derechas, con imagen de costillas cervicales bilaterales con diferentes características anatómicas. Con este caso se discute cómo una variante de la anatomía produce patología y cuando no está relacionada a compresión.
\end{abstract}

Palabras clave: Costilla. Cervical. Plexo braquial. Compresión.

\begin{abstract}
Background: The cervical rib can be a rare finding in asymptomatic subjects. When it produces compression, it affects variably the vasculo-nervous structures of the brachial plexus; the clinical history is progressive. The imaging studies indicate the site of osteo-neuro-vascular conflict and electrophysiological studies indicate the degree of neural involvement. Case report: We present a patient with an eight-month history of paresis and right dysesthesias, with an image of bilateral cervical ribs with different anatomical characteristics. This case discusses how a variant of the anatomy produces pathology and when it is not related to compression.
\end{abstract}

Key words: Rib. Cervical. Brachial plexus. Compression.

\section{Introducción}

La costilla cervical o costilla supernumeraria de Eve (OMIM:117900) es una costilla accesoria que se articula con las apófisis transversas de C5 o C6. Es una variable anatómica con una prevalencia diversa, dependiendo de las diferentes poblaciones, entre el $0.5 \%$ y el $7 \%$. El $90 \%$ de los casos son asintomáticos. La prevalencia aumenta en sujetos con tumores de las crestas neurales y mesenquimales (23-33\%), y se asocia en un $30 \%$ de los pacientes con síndrome del opérculo cervicotorácico (SOCT) $)^{1,2}$. Los síntomas y 
los signos son vasculonerviosos por compresión del segmento supraclavicular del plexo braquial, con afección sobre todo del tronco inferior; en otras ocasiones menos frecuentes la compresión es infraclavicular a los fascículos posterior y medial del plexo braquial. El lugar del conflicto neurovascular y óseo (CONV) es el pasadizo donde el plexo cambia su orientación de horizontal a vertical ${ }^{3-5}$. Su patogenia tiene bases embriológicas y con fenotipos en poblaciones con pleuroapófisis vestigiales, «recuerdos evolutivos"; en otros casos se ha relacionado con mutaciones en los genes HOX y la desactivación del factor de diferenciación del crecimiento 11 en la cuarta semana de embriogénesis, resultando en una falla en la diferenciación de los esclerotomas, por lo que se considera como una anomalía congénita ${ }^{6}$. Afecta mayoritariamente a mujeres (2:1). La sintomatología más común es la cervicobracalgia, acompañada de parestesias y disminución de la fuerza de los músculos de la región tenar, y compromiso de los territorios de los nervios ulnar y mediano. La evolución clínica es lenta. Al ser una patología ósea, el diagnóstico se fundamenta en la reconstrucción tomográfica, los estudios electrofisiológicos y las neurografías por resonancia, para constatar el grado de afección del plexo braquial y su potencial de recuperación $n^{3,5}$. Las características, las asociaciones y las condiciones para que una variable de la normalidad produzca enfermedad se discuten a continuación, en el contexto de un paciente con costillas cervicales bilaterales en el que únicamente existe patología unilateral.

\section{Caso clínico}

Varón de 27 años que acude a la consulta del Hospital José Carrasco Arteaga por un cuadro de 8 meses de evolución de sensación de parestesias y disestesia (HP:0003401, HP:0012534) en los dermatomas $\mathrm{C} 7$ y $\mathrm{C} 8$ del lado derecho. Presenta prominentes pliegues tenares y palmares proximales («mano simiana", HP: 0000954), disminución de la fuerza en el grupo de los músculos lumbricales (HP: 0002460) y en la flexión de los dedos, signo de Fromet positivo, prueba de provocación de Roos positivo con caída del miembro superior derecho, y prueba de Adson y Wright positiva con disminución del pulso radial derecho. El estudio de electroconducción reveló una disminución del reclutamiento de las fibras: primer interóseo dorsal derecho, extensor del índice derecho, normalidad de los grupos proximales del miembro superior y de los flexores y extensores comunes de los dedos, y hallazgos de afección del tronco inferior del plexo braquial derecho; el lado izquierdo no muestra síntomas ni signos en la exploración física, y la electroconducción indicó normalidad. La imagen demostró costillas cervicales: derecha con el extremo distal libre e izquierda con su extremo distal articulado en la cara posterior del tubérculo del músculo escaleno anterior de la primera costilla. En el estudio vascular se observó una disminución del calibre de la vena axilar derecha en el lugar del CONV (Fig. 1).

Se realizó la intervención quirúrgica de exéresis de costilla cervical con abordaje supraclavicular. La costilla cervical derecha estaba articulada a la apófisis transversa C6, con el borde libre estrechando el opérculo cervicotorácico, y el tronco nervioso inferior del plexo braquial derecho estaba comprimido y deformado. Se realizaron neurólisis y adeherenciólisis del lugar del conflicto con transposición, liberación del segmento comprimido y fresado del borde superior de la primera costilla (Fig. 2).

A los 3 meses el paciente tuvo mejoría sintomática y con rehabilitación física mejoró la fuerza de aprehensión palmar; sin embargo, persistió con valores de reclutamiento bajos en los grupos musculares distales, sin sintomatología ni alteración en la electroconducción en el lado izquierdo.

\section{Discusión}

La segunda forma más frecuente de costilla cervical es la bilateral. La costilla cervical unilateral derecha es la más presente. De acuerdo con el tamaño y el desarrollo, una costilla cervical puede ser: I) completa con su borde distal articulado; II) incompleta con su extremo distal libre; III) incompleta con banda fibrosa que la une al tórax; y IV) como banda ósea corta que se puede confundir con una apofisomegalia transver$\mathrm{sa}^{3-7}$. Las características anatómicas que pueden aumentar la posibilidad de compresión neurovascular aún no están completamente esclarecidas, pero el borde libre verdadero que en el movimiento oblitera las estructuras del opérculo es una teoría fuertemente sustentada. Cuando en la imagen se distingue el borde libre y el paciente está asintomático, seguramente la posibilidad más explicable es que se trate de una articulación fibrosa en el extremo libre que estabilice la costilla accesoria, ya que este tejido fibroso no se distingue en las imágenes de reconstrucción ósea ${ }^{8}$. La sintomatología más habitual y más consultada por el paciente es el SOCT neural; sin embargo; es una condición sine qua non que las estructuras vasculares 

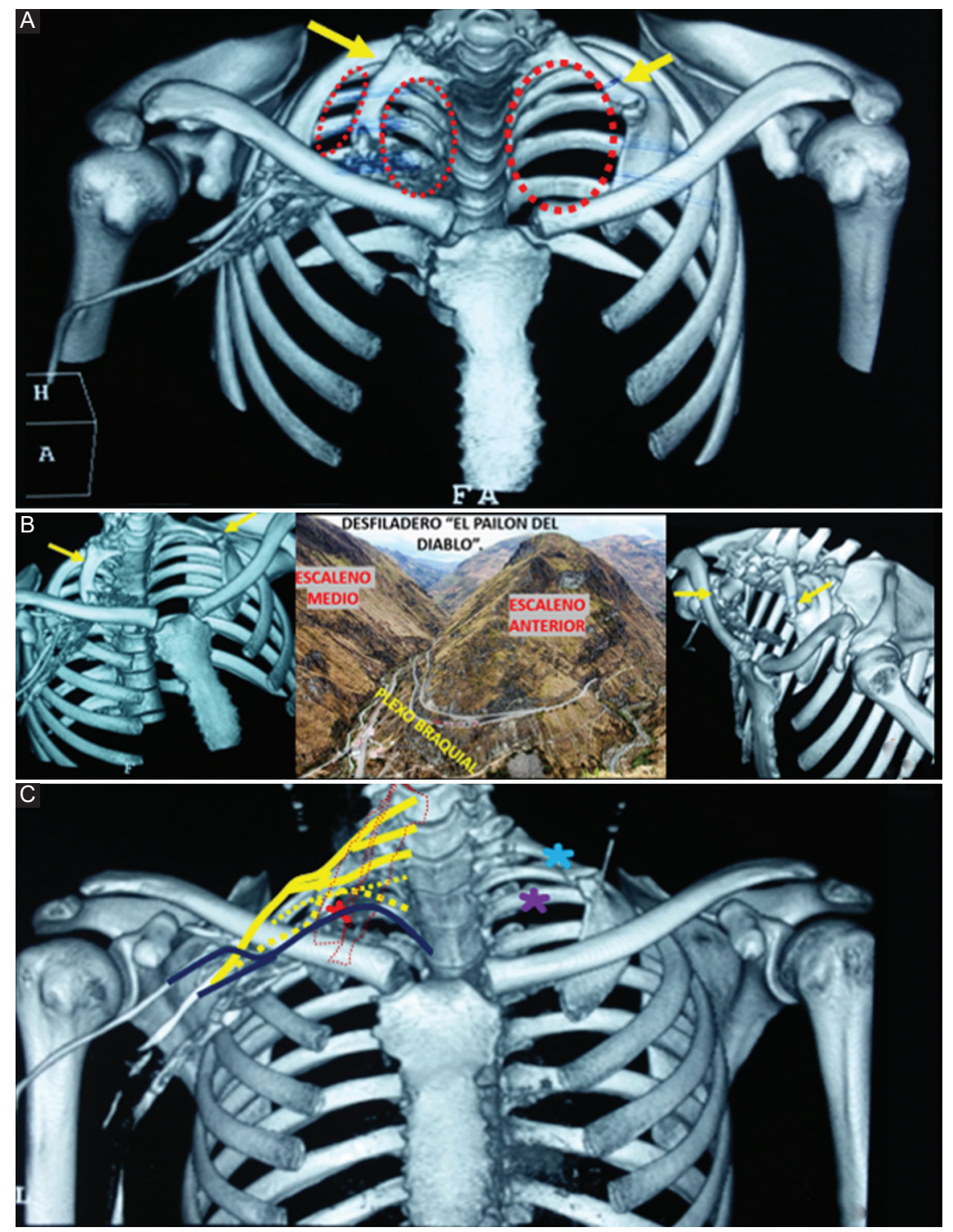

Figura 1. Características de las costillas cervicales. A: imagen de reconstrucción tomográfica tridimensional ósea y angiotomografía venosa derecha. Comparación de los opérculos torácicos (líneas punteadas rojas), el derecho dividido en dos y el izquierdo sin división, y costillas accesorias (flechas). B: costillas accesorias (flechas); entre las tomografías, foto del desfiladero El Pailón del Diablo, en Baños, Tungurahua, Ecuador, que ejemplifica la anatomía cervicotorácica. C: plexo braquial derecho (líneas amarillas), tronco inferior del plexo braquial derecho (líneas punteadas amarillas), músculos escaleno anterior y posterior (líneas punteadas rojas), vena axilar (línea azul), conflicto osteoneurovascular derecho (asterisco rojo), opérculo cervicotorácico izquierdo libre (asterisco púrpura) y borde distal izquierdo articulado a la primera costilla (asterisco cardenillo).

estén comprometidas 9 . Un aspecto importante en el diagnóstico de exclusión es la presencia de signos de claudicación vascular, o las llamadas pruebas de provocación, que tienen mayor sensibilidad (pruebas de Adson, Falconner y Weddel, y Wright) y lo diferencian de otras plexopatías: En el caso que presentamos, el paciente consultó por un cuadro de cervicobracalgia derecha con evidencia de neuropatía del tronco inferior del plexo braquial, y la imagen demostró en el lado derecho la costilla accesoria con borde libre, lugar del CONV (tipo II), mientras que en el lado izquierdo, sin sintomatología, la costilla accesoria es de tipo I. La exploración física demostró con las pruebas de provocación compromiso vascular, por lo que es un dato importante de SOCT arterial y neural.

La evolución natural es lenta y se requiere un estrés repetido para presentar sintomatología. Por ser una patología congénita, el tiempo de diagnóstico es tardío. Es más frecuente en los adultos de mediana edad, lo que demuestra el fenómeno de estrés 


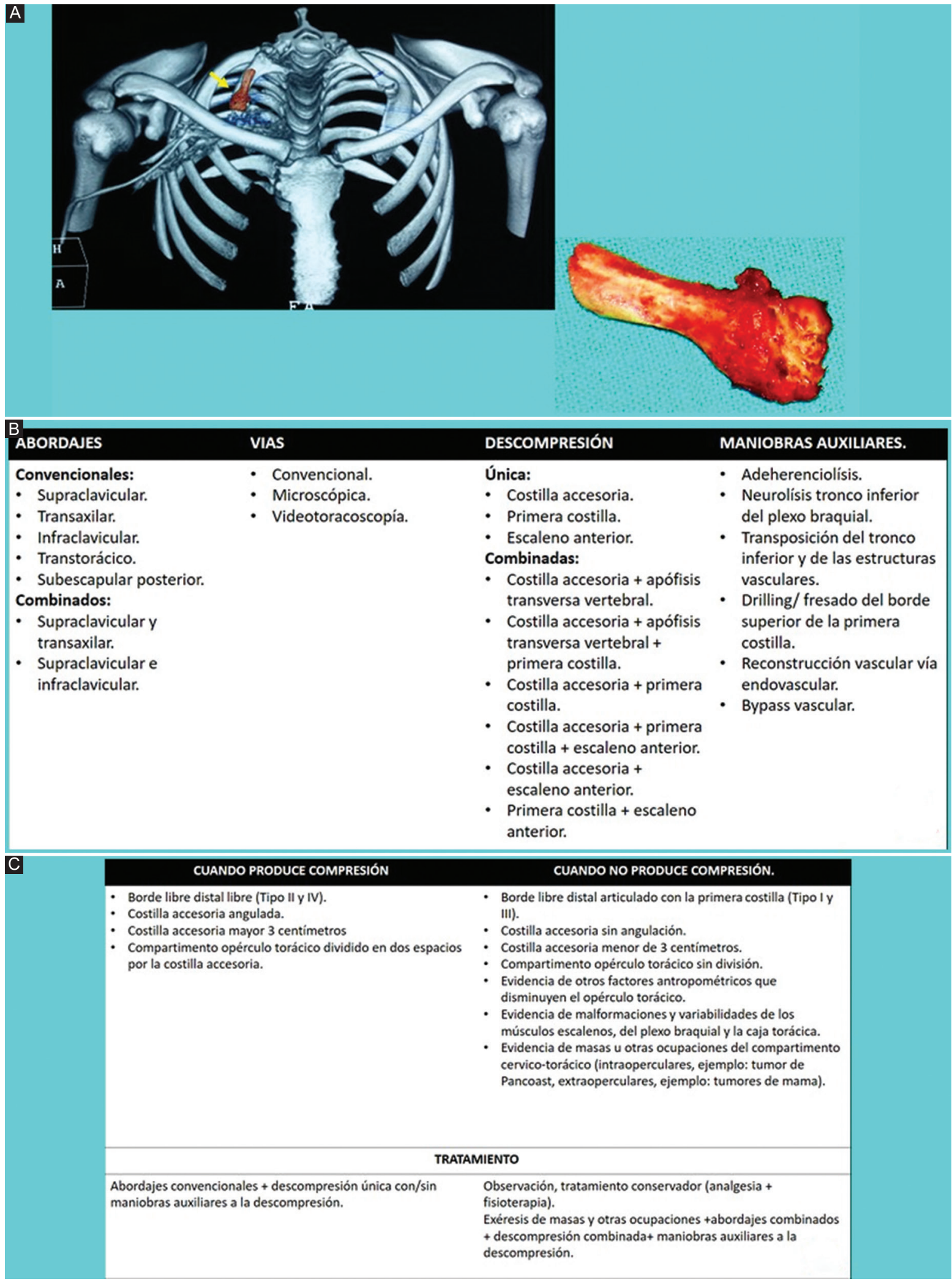

Figura 2. Espécimen de costilla accesoria derecha y tablas que resumen las consideraciones en cirugía de compresión cervicotorácica por costilla accesoria. A: espécimen del extremo distal de la costilla accesoria, que fue resecada en dos partes, y su ubicación en la tomografía en reconstrucción ósea tridimensional. B: variantes y opciones de cirugía para costilla accesoria. C: resumen y comparación de los escenarios y los tratamientos según si la costilla accesoria produce compresión o no. 
prolongado para reducir y atrapar el compartimento cervicotorácico ${ }^{2,9}$. El daño nervioso muchas veces es tipo neuropraxia. Tras el tratamiento tiene buen pronóstico, pero la presencia de atrofia de músculos distales es un signo de recuperación más lenta o incluso incompleta ${ }^{5}$. Los estudios electrofisiológicos, como la velocidad de conducción, pueden ser una herramienta diagnóstica; existen criterios quirúrgicos con evidencia de valores menores de $60 \mathrm{~m} / \mathrm{s}^{9}$. La electromiografía sirve para descartar otras causas y puede predecir la posibilidad de recuperación, sobre todo la latencia de la onda f. En cuanto a la imagen, la neurografía por resonancia magnética del plexo braquial puede mostrar amputación de la fibras. que correspondería a un compromiso avanzado ${ }^{5}$. En nuestro caso hubo signos de atrofia distal, no se evidenció deformidad como mano en garra o de predicador, y los hallazgos intraoperatorios no mostraron cambios permanentes del plexo. A los 3 meses el paciente tuvo mejoría clínica, aunque los estudios electrofisiológicos aún estaban afectados, lo que demuestra que un uso temprano posquirúrgico no es un buen predictor de evolución ni de pronóstico.

Los estadios avanzados de compresión afectan al componente vascular. Las afecciones tardías, como pseudoaneurismas y trombosis, pueden observarse en estudios vasculares como la angiografía y la angiotomografía, e incluso en la ecografía endovascular para detección de estenosis ${ }^{9,10}$. Estas son condiciones que ensombrecen el pronóstico y la recuperación. En nuestro caso hubo concurrencia de claudicación nerviosa y vascular; en el intraoperatorio se encontraron las estructuras indemnes.

Las características anatómicas de vulnerabilidad para el CONV independientes de la presencia de costilla cervical mencionadas por la literatura son el tronco superior del plexo braquial en posición anterior al músculo escaleno anterior, el tronco superior o inferior que atraviese los vientres musculares del escaleno anterior y medio, el músculo escaleno mínimo y accesorio que comprime los troncos y cordones del plexo, bandas de tejido ligamentoso o fibrofascial desde la parte posterior de la primera costilla hasta las inserciones en el tórax, tronco inferior y nervio frénico accesorio que pasa por delante de la vena subclavia antes de girar hacia el pecho, y clavícula bífida; todas ellas son difíciles de distinguir en los estudios de imagen ${ }^{11}$, pueden estar coexistiendo con la costilla cervical y se demuestran intraoperatoriamente. En cuanto la asociación entre ellas, no está descrita, ni son consistentemente causas de la compresión; por lo tanto, la adeherenciólisis y la transposición de estructuras son maniobras que deben acompañar a los procedimientos de exéresis de costillas accesorias, las mismas que fueron practicadas en nuestro caso.

Otros estudios de la anatomía y la conformación de la caja torácica también pueden aumentar la posibilidad de compresión independientemente de la presencia de una costilla accesoria: espacio costoclavicular estrecho en elevación del miembro superior, escoliosis cervicodorsal, gran grosor del músculo subclavio y disminución de la distancia entre el borde del músculo pectoral menor y el revestimiento posterior axilar, e hipertrofia mamaria. Se han evidenciado diferencias estadísticamente significativas de estas variables en sujetos sintomáticos frente a sujetos asintomáticos, pero los estudios tienen limitaciones en describir las posiciones y las maniobras usadas para estandarizar la medición ${ }^{8,11}$; son asociaciones antropométricas que deben ser valoradas en planificación quirúrgica y que pueden ser parte del éxito posquirúrgico. En nuestro paciente no se constataron alteraciones antropométricas visibles.

Otros artículos citan el estrés psicosocial y los síndromes miofasciales en pacientes con diagnóstico de SOCT y sintomatología persistente posterior a la exéresis de la costilla accesoria. En cuanto al dolor, al ser de tipo neuropático, la evolución mayor de 12 meses de dolor prequirúrgico es suficiente para producir fenómenos de centralización, influyendo en que el paciente mantenga un dolor crónico posoperatorio ${ }^{2,8,11}$. Nuestro paciente acudió con dolor, pero no fue el motivo de consulta, pues tuvieron mayor relevancia los síntomas motores, y no evolucionó con dolor neuropático crónico.

Las variaciones del plexo braquial son excepcionales y no está reportada su relación con SOCT y costilla cervical. Los plexos braquiales tipos posfijados (con raíces desde C5/C6 a T2) y con comunicación T2 son los más inferiores y más inclinados, y por ende con mayor posibilidad de obturarse por una costilla cervical. En nuestro caso no se halló variabilidad en la situación del plexo braquial ${ }^{12}$.

La costilla cervical tiene naturaleza congénita. Su demostración en los varones es en la edad adulta temprana, relacionada con la edad de máxima actividad laboral (militares, trabajos con carga de peso, trabajo de oficina) y física (movimientos repetitivos y deportes como natación, básquet, vóley y tenis). También se observa en mujeres de mediana edad con afecciones y pérdida de masa muscular ${ }^{13}$. El caso que presentamos obedece al primer pico de edad en hombres. 
EI SOCT y la costilla cervical con características de compresión tienen como tratamiento principal la descompresión quirúrgica. Este objetivo se puede lograr con el retiro de la primera costilla, el músculo escaleno anterior, la costilla accesoria o una combinación de ellas; la resección de las dos primeras y sus combinaciones aumentan el tiempo operatorio, el riesgo anestésico, los días de hospitalización y el tiempo de recuperación, sin que haya diferencia en cuanto a mejores resultados funcionales frente a la resección única de la costilla cervical accesoria con apófisis transversa vertebral distal. Esta última se ha asociado a mejoría posquirúrgica de la funcionalidad en las pruebas de Roos y de estrés por elevación del brazo, y a seguridad en cuanto a complicaciones. Los abordajes mencionados en la literatura son el supraclavicular, que ofrece visualización y control del plexo braquial y de la arteria subclavia; el transaxilar, que permite la resección de la primera costilla y la visualización de la arteria axilar; y menos usados, el subescapular posterior, el transtorácica y los combinados (supraclavicular con transaxilar y supraclavicular con infraclavicular, para casos de recidivas, costillas cervicales grandes y asociaciones a aneurismas) ${ }^{14,15}$. La vía puede ser convencional microquirúrgica y en centros de experiencia por videotoracoscopia, que trae mayor dificultad en la luxación de la primera costilla, sobre todo en pacientes con gran musculatura ${ }^{16}$. Como mencionamos, según los hallazgos intraoperatorios se pueden observar distintas patologías que son concurrentes a la presencia de la primera costilla, y por ello se mencionan procedimientos auxiliares como adherenciólisis y neurólisis del plexo braquial, que son usados en presencia de bandas fibromusculares y ligamentarias anexas en casos de recidivas. En caso de tronco inferior deformado y desplazado, son necesarias la liberación y la transposición neural. Además, se mencionan el fresado y la exéresis parcial de la primera costilla ${ }^{14-18}$. Finalmente, el compromiso vascular exige complemento de manejos tales como el endovascular y bypass vasculares para la resolución de pseudoaneurismas y trombosis s,17,18. $^{9}$.

En nuestro caso utilizamos el abordaje supraclavicular y realizamos la exéresis de la costilla cervical en dos partes; la última incluyó la cabeza y parte de la apófisis transversa vertebral. Visualizamos las estructuras del plexo braquial indemnes, pero deformadas, por lo que procedimos a realizar adherenciólisis, liberación, neurólisis y transposición del tronco inferior para aumentar la posibilidad de éxito posoperatorio y evitar las recidivas.

\section{Conclusiones}

La costilla cervical es una variable de la normalidad que debe reunir condiciones anatómicas y asociaciones para producir enfermedad. Los escenarios posibles son: sujetos asintomáticos que se someten a exámenes de imagen con evidencia de esta variabilidad, otros que tengan plexopatía y el origen de esta sea la compresión directa por una costilla cervical, y por último individuos con plexopatía y costilla cervical con otros factores que estrechen el opérculo cervicotorácico e indirectamente produzcan compresión con la costilla accesoria. Es necesaria la individualización de los casos para tomar la decisión de una intervención quirúrgica adecuada. Además, los hallazgos intraoperatorios determinan las decisiones que garanticen el éxito posoperatorio. En la actualidad, el manejo de esta patología no tiene un único objetivo de descompresión, sino también de prevenir la recidiva y tratar los factores asociados. En este paciente, la sintomatología fue producida por la costilla cervical derecha con características de compresión, mientras que en el lado izquierdo la costilla cervical no tiene características de producir patología. Es un caso que ilustra la coexistencia de una condición que puede tener dualidad en ser una variante anatómica de la normalidad y a la vez una patología que requiere tratamiento de descompresión y maniobras que garanticen el éxito posquirúrgico.

\section{Financiamiento}

Esta investigación no recibió subvenciones específicas de agencias de sectores público, comercial o con fines de lucro.

\section{Conflictos de intereses}

Los autores declaran no tener conflicto de intereses.

\section{Responsabilidades éticas}

Protección de personas y animales. Los autores declaran que para esta investigación no se han realizado experimentos en seres humanos ni en animales.

Confidencialidad de los datos. Los autores declaran que han seguido los protocolos de su centro de trabajo sobre la publicación de datos de pacientes. 
Derecho a la privacidad y consentimiento informado. Los autores han obtenido el consentimiento informado de los pacientes y/o sujetos referidos en el artículo. Este documento obra en poder del autor de correspondencia.

\section{Bibliografía}

1. Schumacher R, Mai A, Gutjahr P. Association of rib anomalies and malignancy in childhood. Eur J Pediatr. 1992;151:432-4.

2. Urschel HC, Kourlis H. Thoracic outlet syndrome: a 50-year experience at Baylor University Medical Center. Proceedings (Baylor University Medical Center). 2007;20:125-35.

3. Atasoy E. Thoracic outlet syndrome: anatomy. Hand Clinics. 2004;20:7-14

4. Abimbola EO, Willido AA. Prevalence of cervical ribs in a Nigerian population. J Med Dental Sci. 2014;13:5-7.

5. Henry BM, Vikse J, Sanna B, Taterra D, Gomulska M, Pękala PA, et al. Cervical rib prevalence and its association with thoracic outlet syndrome: a meta-analysis of 141 studies with surgical considerations. World Neurosurg. 2018;110:e965-78.

6. Ten Broek CMA, Bakker AJ, Varela-Lasheras I, Bugiani M, van Dongen $S$, Galis F. Evo-devo of the human vertebral column: on homeotic transformations, pathologies and prenatal selection. Evol Biol. 2012;39:456-71.

7. Torres Cueco R. La columna cervical: síndromes clínicos y su tratamiento manipulativo. Madrid: Médica Panamericana; 2006.
8. Sharma DK, Vishnudutt, Sharma V, Rathore M. Prevalence of 'cervical rib' and its association with gender, body side, handedness and other thoracic bony anomalies in a population of Central India. Indian Journal of Basic and Applied Medical Research. 2014;3:593-7.

9. Urschel HC Jr, Razzuk MA. Neurovascular compression in the thoracic outlet: changing management over 50 years. Ann Surg. 1998;228:609-17.

10. Kim TI, Sarac TP, Orion KC. Intravascular ultrasound in venous thoracic outlet syndrome. Ann Vasc Surg. 2019:54:118-22.

11. Leonhard V, Smith R, Caldwell G, Smith HF. Anatomical variations in the brachial plexus roots: implications for diagnosis of neurogenic thoracic outlet syndrome. Ann Anat. 2016;206:21-6.

12. Pellerin M, Kimball Z, Tubbs RS, Nguyen S, Matusz P, Cohen-Gadol AA, et al. The prefixed and postfixed brachial plexus: a review with surgical implications. Surg Radiol Anat. 2010;32:251-60.

13. Levine NA, Rigby BR. Thoracic outlet syndrome: biomechanical and exercise considerations. Healthcare (Basel). 2018;6:68.

14. Morel J, Pirvu A, Elie A, Gallet N, Magne JL, Spear R. Functional results of cervical rib resection for thoracic outlet syndrome: impact on professional activity. Ann Vasc Surg. 2019;56:233-9.

15. Umerani MS, Ashqan Y, Burhan H, Darwish AAQ, Mohammed HR, Alhaji Z. Excision of cervical rib through supraclavicular approach. J Rare Disord Diagn Ther. 2017;3:10.

16. Nuutinen H, Riekkinen $T$, Aittola V, Mäkinen K, Kärkkäinen JM. Thoracoscopic versus transaxillary approach to first rib resection in thoracic outlet syndrome. Ann Thorac Surg. 2018;105:937-42.

17. Maqbool T, Novak CB, Jackson T, Baltzer HL. Thirty-day outcomes following surgical decompression of thoracic outlet syndrome. Hand (N Y). 2019;14:107-13.

18. Jubbal KT, Zavlin D, Harris JD, Liberman SR, Echo A. Morbidity of first rib resection in the surgical repair of thoracic outlet syndrome. Hand (N Y). 2019;14:636-40 